\title{
Synthesis and antimicrobial activity of new triazolopyrimidinecarbonitrile derivatives
}

\author{
Azza M. Kadry, ${ }^{*}$ Eatedal H. Abdel Aal, Hanan A. Abdel-Fattah, and Amany M. Al- \\ Mahmoudy
}

\author{
Pharmaceutical Organic Chemistry Department, Faculty of Pharmacy \\ Zagazig University, Zagazig, Egypt \\ E-mail: azzamokadry@yahoo.com
}

\section{Dedicated to Professor Nouria A. Al-Awadi on the occasion of her 55th birthyear, for her scientific achievements and on the occasion of her appointment as Vice-President for Academic Affairs, Kuwait University}

\begin{abstract}
5-Cyano-2-hydrazino-3-methyl-6-(3,4,5-trimethoxyphenyl)pyrimidin-4(3H)-one was prepared and reacted with carbon disulphide to give the corresponding 3-thioxo derivative 2 . Hydrazinolysis of $\mathbf{2}$ gave the 3-hydrazino derivative 3. Reaction of 3 with aromatic aldehydes, formic acid, acid chlorides, carbon disulfide, nitrous acid, pyruvic acid, acetylacetone and benzoylacetone gave the corresponding condensation products 4a-g, 5, 6a-b, 7, 8, 9 and 10a-b. Some of the newly synthesized compounds exhibited a high antimicrobial activity against both gram positive and gram negative bacteria and fungi.
\end{abstract}

Keywords: Pyrimidin-4(3H)-ones, [1,2,4]triazolo[4,3-a]pyrimidin-7-ones, pyrimido$[1,2: 4,3][1,2,4]$ triazolo[5,1-c] $[1,2,4]$ triazine, triazolo[3,4:5,1][1,2,4]triazolo[4,3-a] pyrimidin$7(6 H)$-one, antimicrobial activity

\section{Introduction}

Many compounds containing the pyrimidine nucleus are of significant biological importance and are used as antibacterial, ${ }^{1-5}$ antifungal, ${ }^{6,7}$ antimalarial, ${ }^{8}$ antitumour, ${ }^{9-11}$ antiviral, ${ }^{12-15}$ antiinflammatory ${ }^{16-18}$ or antihypertensive ${ }^{19-21}$ agents. In this work, we synthesized some new 3,4,5-trimethoxyphenyltriazolopyrimidinecarbonitrile derivatives in the hope of obtaining new products of superior biological activity. 


\section{Results and Discussion}

The starting 6-cyano-2,3-dihydro-8-methyl-3-thioxo-5(3,4,5-trimethoxyphenyl)[1,2,4]-triazolo [4,3-a]pyrimidin-7 $(8 \mathrm{H})$-one 2 was prepared accordingly to the procedure reported by Geies et al. $^{22}$ Thus, condensation of $\mathbf{1}$ with carbon disulphide in ethanol in the presence of KOH gave 2 in $56 \%$ yield.

The 3-hydrazino derivative 3, was obtained by heating 2 with hydrazine hydrate. Condensation of the latter with different aromatic aldehydes namely, benzaldehyde, 2chlorobenzaldehyde, 4-hydroxybenzaldehyde, 4-methoxybenzaldehyde, 4-nitrobenzaldehyde, 2fluorobenzaldehyde and 4-N,N-dimethylaminobenzaldehyde afforded the corresponding 3arylidenehydrazino-6-cyano-8-methyl-5-(3,4,5-trimethoxyphenyl)[1,2,4]triazolo[4,3-a]

pyrimidin-7 $(8 H)$-ones 4 a-g respectively.

Cyclocondensation of compound 3 with formic acid gave 8-cyano-6-methyl-9-(3,4,5trimethoxyphenyl)triazolo[3,4:5,1][1,2,4]triazolo[4,3-a]pyrimidin-7(8)-one 5 in $40 \%$ yield. One the ather hand, the 3-methyl and 3-phenyl derivatives 6a-b were prepared by the reaction of acetyl chloride or benzoyl chloride with 3.

Condensation of 3 with carbon disulfide in absolute ethanol in the presence of $\mathrm{KOH}$ gave 8cyano-6-methyl-2,3-dihydro-3-thioxo-9(3,4,5-trimethoxyphenyl)triazolo[3,4:5,1][1,2,4] triazolo [4,3-a]pyrimidin-7(6H)-one 7 in $30 \%$ yield.

Treatment of compound 3 in $2 \mathrm{~N}$ hydrochloric acid at $10{ }^{\circ} \mathrm{C}$ with a solution of sodium nitrite, gave the corresponding tetrazolotriazolopyrimidin-7-one 8 in 55\% yield.

Moreover condensation of compound 3 with pyruvic acid by heating at $180{ }^{\circ} \mathrm{C}$ gave the corresponding pyrimido[1,2:4,3][1,2,4]triazolo[5,1-c][1,2,4]triazine-4,8-dione 9 in 47\% yield.

Condensation of compound $\mathbf{3}$ with the appropriate $\beta$-dicarbonyl compounds; namely, acetylacetone and benzoylacetone gave the corresponding pyrazol-1-yl[1,2,4]triazolopyrimidin7-ones 10a-b.

The in vitro antimicrobial activity of the tested compounds summarized in Table 1 revealed the following: compound $\mathbf{8}$ was effective against gram positive cocci (S. aureus), gram negative bacilli (B. subtilis), and gram negative rods (E. coli and C. albicans). Compound 10a showed marked antibacterial activity but did not show any antifungal activity. Compound $\mathbf{9}$ was active only against gram negative bacilli (B. subtilis). Compound 7 did not show any antibacterial activity but showed marked antifungal activity. Compound 2 was inactive against $\mathrm{S}$. aureus but showed marked activity against B. subtilis, E. coli and C. albicans. Compound $\mathbf{3}$ was active only against S. aureus and B. subtilis but showed no antifungal activity. Compound 4d was active only against $\mathrm{S}$. aureus and E. coli. Compound $4 \mathbf{e}$ was active only against St. aureus and showed marked antifungal activity. Compound 5 was completely inactive. 


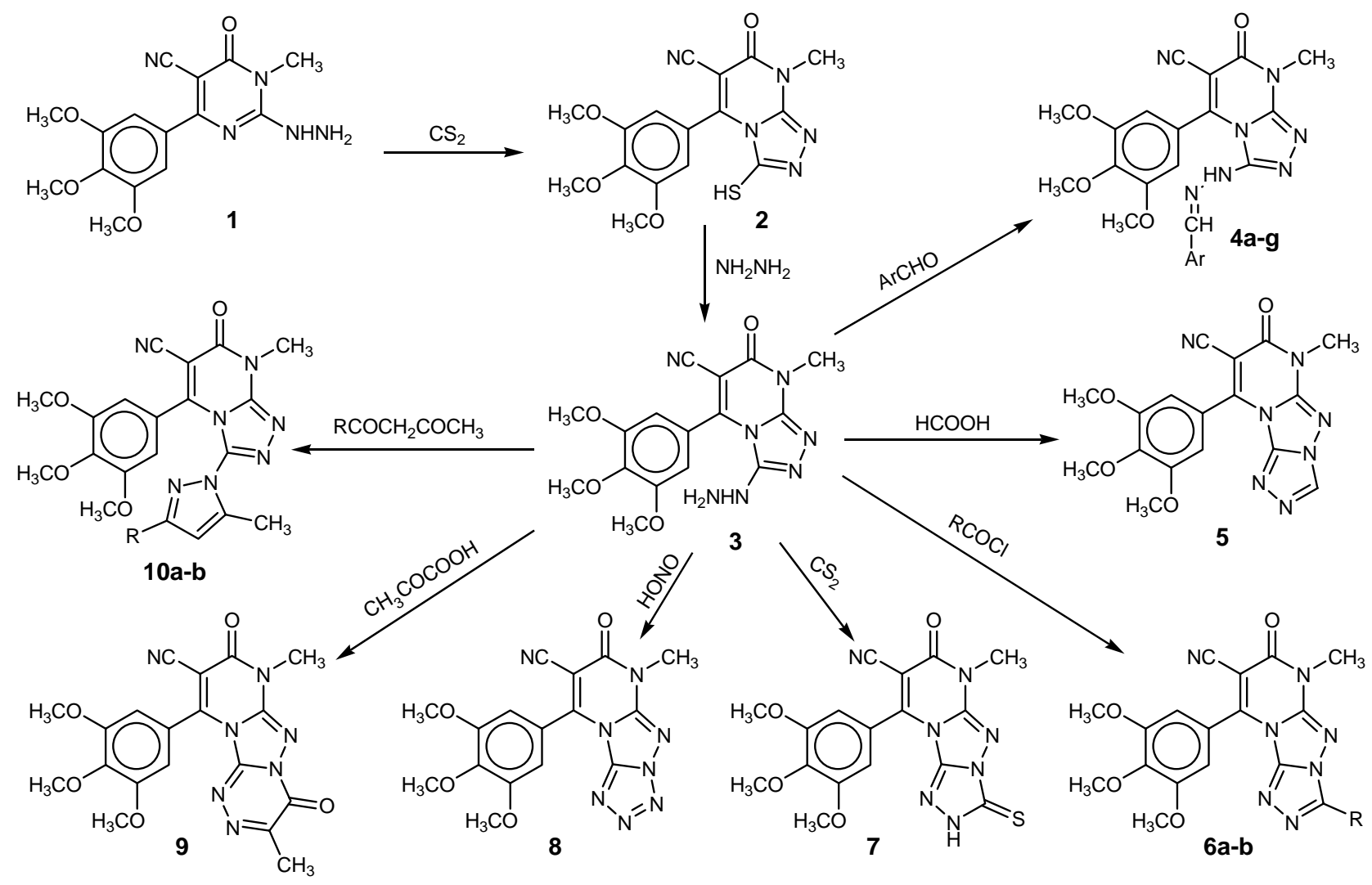

Table 1. Antimicrobial activity

\begin{tabular}{ccccc}
\hline \multirow{2}{*}{ Compound No. } & \multicolumn{4}{c}{ Inhibition zone diameter $(\mathrm{mm})$} \\
\cline { 2 - 5 } & S. aureus & B. subtilis & E. coli & C. albicans \\
\hline $\mathbf{2}$ & - & 16 & 14 & 18 \\
$\mathbf{3}$ & 11 & 8 & - & - \\
$\mathbf{4 d}$ & 10 & - & 14 & - \\
$\mathbf{4 e}$ & 8 & - & - & 10 \\
$\mathbf{5}$ & - & - & - & - \\
$\mathbf{6 b}$ & - & 19 & 22 & - \\
$\mathbf{7}$ & - & - & - & 20 \\
$\mathbf{8}$ & 17 & 22 & 13 & 20 \\
$\mathbf{9}$ & - & 12 & - & - \\
$\mathbf{1 0 a}$ & 17 & 10 & 18 & - \\
Trimethoprim & 19 & 19 & 21 & - \\
\hline
\end{tabular}

(-) No inhibition zone. 


\section{Experimental Section}

General Procedures. Melting points were determined on a Gallenkamp Digital melting point apparatus and are uncorrected. IR spectra were determined as $\mathrm{KBr}$ discs on a Shimadzu FTIR 8000 Spectrophotometer. ${ }^{1} \mathrm{H}$ NMR spectra were measured using Varian Gemini-200, $200 \mathrm{MHz}$ spectrometers. Mass spectra (EIMS) were run on Hewlett Packard 5988 spectrometer. Elemental analyses were carried out at the Microanalytical Center, Faculty of Science, Cairo University, Cairo, Egypt and the Microanalytical Center, National Research Center, Dokki, Egypt. Progress of the reactions was monitored by TLC using sheets precoated with UV fluorescent silica gel Merck 60 F254. Compound 1 was prepared as reported. ${ }^{23}$

\section{6-Cyano-2,3-dihydro-8-methyl-3-thioxo-5-(3,4,5-trimethoxyphenyl)[1,2,4]triazolo-[4,3-a]} pyrimidin-7(8H)-one (2). To an ice cooled solution of $1(0.66 \mathrm{~g}, 2 \mathrm{mmol})$ in absolute ethanol $(10 \mathrm{~mL})$ containing potassium hydroxide $(0.11 \mathrm{~g}, 2 \mathrm{mmol})$, carbon disulfide $(1 \mathrm{~mL})$ was added dropwise with stirring. The mixture was diluted with absolute ethanol $(10 \mathrm{ml})$ and was refluxed for $6 \mathrm{~h}$. The reaction mixture was filtered, concentrated, diluted with water and neutralized with acetic acid. The precipitated product was crystallized from dioxane as yellow crystals, mp 225$257^{\circ} \mathrm{C}$; yield $0.37 \mathrm{~g}(56 \%)$. IR: 2939, $2838(\mathrm{CH}$ aliphatic), $2542(\mathrm{SH}), 2231(\mathrm{C} \equiv \mathrm{N}), 1685(\mathrm{C}=\mathrm{O})$ $\mathrm{cm}^{-1} .{ }^{1} \mathrm{H}$ NMR $\left(\mathrm{CDCl}_{3}\right): \delta 3.69-3.97\left(\mathrm{~m}, 12 \mathrm{H}, 3 \mathrm{OCH}_{3}, \mathrm{NCH}_{3}\right), 7.08-7.15(\mathrm{~m}, 2 \mathrm{H}, \mathrm{ArH}), 8.70$ (br, $1 \mathrm{H}$, exchangeable). Anal. calcd. for $\mathrm{C}_{16} \mathrm{H}_{15} \mathrm{~N}_{5} \mathrm{O}_{4} \mathrm{~S}$ (373.4): C, 51.47; H, 4.02; N, 18.76\% Found: C, 51.67; H, 4.31; N, 18.93\%

6-Cyano-3-hydrazino-8-methyl-5-(3,4,5-trimethoxyphenyl)[1,2,4]triazolo[4,3-a]pyrimidine7(8H)-one (3). A mixture of $2(0.37 \mathrm{~g}, 1 \mathrm{mmol})$ and hydrazine hydrate $(5 \mathrm{~mL}, 99 \%)$ was heated under reflux for $3 \mathrm{~h}$. The formed precipitate was collected, washed with hot ethanol and crystallized from DMF/ethanol mixture as yellow crystals, m.p $284-286{ }^{\circ} \mathrm{C}$; yield $0.11 \mathrm{~g} \mathrm{(30 \% )}$. IR: $3324\left(\mathrm{NH}, \mathrm{NH}_{2}\right), 2210(\mathrm{C} \equiv \mathrm{N}), 1656(\mathrm{C}=\mathrm{O}) \mathrm{cm}^{-1} .{ }^{1} \mathrm{H}$ NMR (DMSO-d $)$ ): $\delta 3.35\left(\mathrm{~s}, 2 \mathrm{H}, \mathrm{NH}_{2}\right.$, exchangeable), $3.75\left(\mathrm{~s}, 3 \mathrm{H}, \mathrm{NCH}_{3}\right), 3.84\left(\mathrm{~s}, 3 \mathrm{H}, \mathrm{p}-\mathrm{OCH}_{3}\right), 3.85\left(\mathrm{~s}, 6 \mathrm{H}, 2 \mathrm{~m}-\mathrm{OCH}_{3}\right), 7.32(\mathrm{~s}, 2 \mathrm{H}$, ArH), 9.00 (s, $1 \mathrm{H}, \mathrm{NH}$, exchangeable). Anal. calcd. for $\mathrm{C}_{16} \mathrm{H}_{17} \mathrm{~N}_{7} \mathrm{O}_{4}$ (371.32): C, 51.75; H, 4.58; N, 26.42\% Found: C, 52.11; H, 4.22; N, 26.01\%

3-Arylidenehydrazino-6-cyano-8-methyl-5-(3,4,5-trimethoxyphenyl)[1,2,4]triazo-lo[4,3-a] pyrimidin-7(8H)-ones (4a-g). A mixture of $3(0.37 \mathrm{~g}, 1 \mathrm{mmol})$ and the appropriate aromatic aldehyde $(1.1 \mathrm{mmol})$ in glacial acetic acid $(10 \mathrm{ml})$ was heated under reflux for $6 \mathrm{~h}$. After cooling and dilution with water, the formed precipitate was collected and crystallized from acetic acid as yellow crystals of 4a-g (Table 2).

8-Cyano-6-methyl-9-(3,4,5-trimethoxyphenyl)triazolo[3,4:5.1][1,2,4]triazolo[4,3-a]pyrimidin7(6H)-one (5). A solution of $3(0.74 \mathrm{~g}, 2 \mathrm{mmol})$ in formic acid $(15 \mathrm{ml})$ was heated under reflux for $15 \mathrm{~h}$. After cooling and dilution with water, the formed precipitate was collected and crystallized from dioxane as white crystals, mp $260-262{ }^{\circ} \mathrm{C}$; yield $0.3 \mathrm{~g}(40 \%)$. IR: $3005(\mathrm{CH}$ aromatic), 2940, 2838, 2756 ( $\mathrm{CH}$ aliphatic), $2219(\mathrm{C} \equiv \mathrm{N}), 1668(\mathrm{C}=\mathrm{O}) \mathrm{cm}^{-1} .{ }^{1} \mathrm{H}$ NMR $\left(\mathrm{CDC}_{13}\right): \delta$ $3.77\left(\mathrm{~s}, 3 \mathrm{H}, \mathrm{NCH}_{3}\right), 3.79$ (s, $\left.3 \mathrm{H}, p-\mathrm{OCH}_{3}\right), 3.92$ (s, 6H, 2m- $\left.\mathrm{OCH}_{3}\right), 7.12$ (s, $\left.2 \mathrm{H}, \mathrm{ArH}\right), 8.06$ (s, 
$1 \mathrm{H},=\mathrm{CH}$ ). Anal. calcd. for $\mathrm{C}_{17} \mathrm{H}_{15} \mathrm{~N}_{7} \mathrm{O}_{4}$ (381.3): C, 53.54; H, 3.93; N, 25.72\% Found: C, 54.00; $\mathrm{H}, 4.33 ; \mathrm{N}, 25.22 \%$

8-Cyano-6-methyl-3-substituted-9-(3,4,5-trimethoxyphenyl)triazolo[3,4:5,1]-[1,2,4]-triazolo [4,3-a]pyrimidin-7(6H)ones (6). A mixture of $3(0.74 \mathrm{~g}, 2 \mathrm{mmol})$ and the appropriate acid chloride $(20 \mathrm{ml})$ was heated under reflux for $8 \mathrm{~h}$. Excess acid chloride was distilled off under reduced pressure and the residue was washed with hot petroleum ether $\left(50-80{ }^{\circ} \mathrm{C}\right)$. The product was collected and crystallized from DMF as yellow crystals (Table 3 ).

8-Cyano-6-methyl-2,3-dihydro-3-thioxo-9(3,4,5-trimethoxy)triazolo[3,4:5,1]-[1,2,4]triazolo [4,3-a]pyrimidin-7(6H)-one (7). To an ice cold solution of $3(0.74 \mathrm{~g}, 2 \mathrm{mmol})$ and $\mathrm{KOH}(0.11 \mathrm{~g}$, $2 \mathrm{mmol})$ in absolute ethanol $(20 \mathrm{~mL})$ was added dropwise with stirring $\mathrm{CS}_{2}(1 \mathrm{~mL})$. The reaction mixture was then heated under reflux for $12 \mathrm{~h}$ and filtered off, concentrated, diluted with water and neutralized with acetic acid. The precipitated product was collected and crystallized from ethanol as buff crystals, mp 320-322 ${ }^{\circ} \mathrm{C}$; yield $0.25 \mathrm{~g}(30 \%)$. IR: $3329(\mathrm{NH}), 2222(\mathrm{C} \equiv \mathrm{N}), 1690$ $(\mathrm{C}=\mathrm{O}) \mathrm{cm}^{-1} .{ }^{1} \mathrm{H}$ NMR $\left(\mathrm{DMSO}_{-} \mathrm{d}_{6}\right): \delta 3.73\left(\mathrm{~s}, 3 \mathrm{H}, \mathrm{NCH}_{3}\right), 3.75$ (s, 6H, 2m- $\left.\mathrm{OCH}_{3}\right), 3.77$ (s, 3H, $p-$ $\left.\mathrm{OCH}_{3}\right), 6.93(\mathrm{~s}, 2 \mathrm{H}, \mathrm{ArH}), 8.70\left(\mathrm{~s}, 1 \mathrm{H}, \mathrm{NH}\right.$, exchangeable). Anal. calcd. for $\mathrm{C}_{17} \mathrm{H}_{15} \mathrm{~N}_{7} \mathrm{O}_{4} \mathrm{~S}$ (413.4): C, 49.39; H, 3.63; N, 23.73\% Found: C, 48.99; H, 3.20; N, 24.01\%

\section{8-Cyano-6-methyl-9-(3,4,5-trimethoxyphenyl)tetrazolo[1,5:1,5][1,2,4]triazolo[4,3-a]}

pyrimidin-7(6H)-one (8). An aqueous solution of sodium nitrite $(10 \mathrm{~mL}, 5 \%)$ was added dropwise at $10{ }^{\circ} \mathrm{C}$ with stirring over 15 minutes to a mixture of $3(0.18 \mathrm{~g}, 0.5 \mathrm{mmol})$ in $2 \mathrm{~N}$ hydrochloric acid $(15 \mathrm{ml})$. The mixture was allowed to stand at room temperature for $2 \mathrm{~h}$. The formed precipitate was collected, washed with ethanol and crystallized from DMF/ethanol as yellow crystals, mp 242-244 ${ }^{\circ} \mathrm{C}$; yield $0.1 \mathrm{~g}$ (55\%). IR: 3117 (CH aromatic), 2939, $2840(\mathrm{CH}$ aliphatic), $2212(\mathrm{C} \equiv \mathrm{N}), 1679(\mathrm{C}=\mathrm{O}) \mathrm{cm}^{-1} .{ }^{1} \mathrm{H} \mathrm{NMR}\left(\mathrm{CDCl}_{3}\right): \delta 3.48\left(\mathrm{~s}, 3 \mathrm{H}, \mathrm{NCH}_{3}\right), 3.92(\mathrm{~s}, 6 \mathrm{H}$, $\left.2 m-\mathrm{OCH}_{3}\right), 3.93\left(\mathrm{~s}, 2 \mathrm{H}, p-\mathrm{OCH}_{3}\right), 7.42$ (s, $\left.2 \mathrm{H}, \mathrm{ArH}\right)$. Anal. calcd. for $\mathrm{C}_{16} \mathrm{H}_{14} \mathrm{~N}_{8} \mathrm{O}_{4}$ (382.2): C, 50.26; H, 3.66; N, 29.32\% Found: C, 49.88; H, 4.01; N, 29.61\%

\section{9-Cyano-3,7-dimethyl-4H-7,8-dihydro-4,8-dioxo-10-(3,4,5-trimethoxyphenyl)-pyrimido} [1,2:4,3][1,2,4]triazolo[5,1-c][1,2,4]triazine (9). A mixture of $3(0.37 \mathrm{~g}, 1 \mathrm{mmol})$ and pyruvic acid $(1.2 \mathrm{mmol})$ was heated in oil bath at $180{ }^{\circ} \mathrm{C}$ for $1 \mathrm{~h}$. After cooling the formed precipitate was heated with ethanol and the precipitate was collected and crystallized from acetic acid as yellow crystals, mp 316-318 ${ }^{\circ} \mathrm{C}$; yield $0.2 \mathrm{~g}(47 \%)$. IR: $2218(\mathrm{C} \equiv \mathrm{N}), 1707(\mathrm{C}=\mathrm{O}), 1677(\mathrm{C}=\mathrm{O})$ $\mathrm{cm}^{-1} .{ }^{1} \mathrm{H}$ NMR $\left(\mathrm{CDCl}_{3}\right): \delta 2.71\left(\mathrm{~s}, 3 \mathrm{H}, \mathrm{CH}_{3}\right), 3.58\left(\mathrm{~s}, 3 \mathrm{H}, \mathrm{NCH}_{3}\right), 3.92\left(\mathrm{~s}, 9 \mathrm{H}, 3 \mathrm{OCH}_{3}\right), 7.41(\mathrm{~s}$, $2 \mathrm{H}, \mathrm{ArH}$ ). Anal. calcd. for $\mathrm{C}_{19} \mathrm{H}_{17} \mathrm{~N}_{7} \mathrm{O}_{5}$ (423.4): C, 53.90; H, 4.01; N, 23.17\% Found: C, 54.32; H, 3.66; N, 23.47\%

\section{6-Carbonyl-3-(3-methylpyrazol-1-yl)-8-methyl-5-(3,4,5-trimethoxyphenyl)-7,8-dihydro}

$[1,2,4]$ triazolo[4,3-a]pyrimidin-7-ones (10). A mixture of $3(0.37 \mathrm{~g}, 1 \mathrm{mmol})$ and the appropriate $\beta$-dicarbonyl compound $(1 \mathrm{mmol})$ in glacial acetic acid $(15 \mathrm{~mL})$ was refluxed for the appropriate time (Table 4). The reaction mixture was cooled and diluted with water, the formed precipitate was collected and crystallized from dioxane as white crystals (Table 4). 
Table 2. 3-Arylidenehydrazino-6-cyano-8-methy-5-(3, 4 ,5-trimethoxyphenyl)[1,2,4]triazolo[4,3-a]pyrimidin-7(8H)-ones 4a-g

\begin{tabular}{|c|c|c|c|c|c|c|c|}
\hline \multirow{2}{*}{$\begin{array}{l}\text { Compd. } \\
\text { No. }\end{array}$} & \multirow[t]{2}{*}{$\mathrm{Ar}$} & \multirow{2}{*}{$\begin{array}{l}\text { Yield } \\
\%\end{array}$} & \multirow{2}{*}{$\begin{array}{l}{ }^{\mathrm{m}} \cdot \mathrm{p} . \\
{ }^{\circ} \mathrm{C}\end{array}$} & \multirow{2}{*}{$\begin{array}{l}\text { Mol. Form. } \\
\text { (Mol. Wt.) }\end{array}$} & \multicolumn{3}{|c|}{$\begin{array}{l}\text { Analysis (\%) } \\
\text { Calcd./Found }\end{array}$} \\
\hline & & & & & $\mathrm{C}$ & $\mathrm{H}$ & $\mathrm{N}$ \\
\hline $4 a$ & $\mathrm{C}_{6} \mathrm{H}_{5}$ & 40 & $295-7$ & $\begin{array}{l}\mathrm{C}_{23} \mathrm{H}_{21} \mathrm{~N}_{7} \mathrm{O}_{4} \\
(459.4)\end{array}$ & $\begin{array}{l}60.13 \\
60.37\end{array}$ & $\begin{array}{l}4.58 \\
5.00\end{array}$ & $\begin{array}{l}21.35 \\
21.10\end{array}$ \\
\hline $4 b$ & $2-\mathrm{ClC}_{6} \mathrm{H}_{4}$ & 35 & $300-2$ & $\begin{array}{l}\mathrm{C}_{23} \mathrm{H}_{20} \mathrm{~N}_{7} \mathrm{O}_{4} \mathrm{Cl} \\
(493.9)\end{array}$ & $\begin{array}{l}55.98 \\
56.22 \\
\end{array}$ & $\begin{array}{l}4.06 \\
3.89\end{array}$ & $\begin{array}{l}19.87 \\
20.10\end{array}$ \\
\hline 4c & 4- $\mathrm{HOC}_{6} \mathrm{H}_{4}$ & 45 & $310-2$ & $\begin{array}{l}\mathrm{C}_{23} \mathrm{H}_{21} \mathrm{~N}_{7} \mathrm{O}_{4} \\
(475.4)\end{array}$ & $\begin{array}{l}58.10 \\
57.90\end{array}$ & $\begin{array}{l}4.42 \\
3.95 \\
\end{array}$ & $\begin{array}{l}20.63 \\
20.22 \\
\end{array}$ \\
\hline 4d & $4-\mathrm{H}_{3} \mathrm{COC}_{6} \mathrm{H}_{4}$ & 30 & $>330$ & $\begin{array}{l}\mathrm{C}_{24} \mathrm{H}_{23} \mathrm{~N}_{7} \mathrm{O}_{4} \\
(489.5)\end{array}$ & $\begin{array}{l}58.89 \\
59.20\end{array}$ & $\begin{array}{l}4.70 \\
4.22 \\
\end{array}$ & $\begin{array}{l}20.04 \\
19.88\end{array}$ \\
\hline $4 e$ & $4-\mathrm{O}_{2} \mathrm{NC}_{6} \mathrm{H}_{4}$ & 50 & $>330$ & $\begin{array}{l}\mathrm{C}_{23} \mathrm{H}_{20} \mathrm{~N}_{8} \mathrm{O}_{6} \\
(504.4)\end{array}$ & $\begin{array}{l}54.76 \\
54.34 \\
\end{array}$ & $\begin{array}{l}3.97 \\
4.33\end{array}$ & $\begin{array}{l}22.22 \\
21.91\end{array}$ \\
\hline $4 f$ & $2-\mathrm{FC}_{6} \mathrm{H}_{4}$ & 20 & $316-8$ & $\begin{array}{l}\mathrm{C}_{23} \mathrm{H}_{20} \mathrm{~N}_{7} \mathrm{O}_{4} \mathrm{~F} \\
(476.5)\end{array}$ & $\begin{array}{l}57.98 \\
58.33 \\
\end{array}$ & $\begin{array}{l}4.20 \\
4.61 \\
\end{array}$ & $\begin{array}{l}20.59 \\
20.87 \\
\end{array}$ \\
\hline $4 g$ & $4-\mathrm{Me}_{2} \mathrm{NC}_{6} \mathrm{H}_{4}$ & 30 & $>330$ & $\begin{array}{l}\mathrm{C}_{25} \mathrm{H}_{26} \mathrm{~N}_{8} \mathrm{O}_{4} \\
(502.4)\end{array}$ & $\begin{array}{l}59.76 \\
60.20 \\
\end{array}$ & $\begin{array}{l}5.18 \\
5.43\end{array}$ & $\begin{array}{l}22.31 \\
22.70\end{array}$ \\
\hline
\end{tabular}

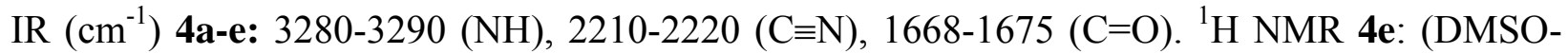
$\left.\mathrm{d}_{6}\right): \delta 3.40\left(\mathrm{~s}, 3 \mathrm{H}, \mathrm{NCH}_{3}\right), 3.77\left(\mathrm{~s}, 3 \mathrm{H}, p-\mathrm{OCH}_{3}\right), 3.84\left(\mathrm{~s}, 6 \mathrm{H}, 2 \mathrm{~m}-\mathrm{OCH}_{3}\right), 7.30(\mathrm{~s}, 2 \mathrm{H}, \mathrm{ArH}), 8.12$ (d, J 8, 2H, ArH), 8.30 (d, J 8 Hz, 2H, ArH), 8.60 (s, 1H, =CH), 11.49 (s, 1H, NH). 4b: MS; m/z $494\left(\mathrm{M}^{+}\right)$.

Table 3. 8-Cyano-6-methyl-3-substituted-9-(3,4,5-trimethoxyphenyl)triazolo[3,4:5,1][1,2,4]triazolo[4,3-a]pyrimidin-7(6H)-ones 6a,b

\begin{tabular}{|c|c|c|c|c|c|c|c|c|}
\hline \multirow{2}{*}{$\begin{array}{l}\text { Compd } \\
\text { No. }\end{array}$} & \multirow[t]{2}{*}{$\mathrm{R}$} & \multirow{2}{*}{$\begin{array}{l}\text { Yield } \\
\%\end{array}$} & \multirow{2}{*}{$\begin{array}{l}\text { Reaction } \\
\text { time }\end{array}$} & \multirow{2}{*}{$\begin{array}{c}\text { m.p. } \\
{ }^{\circ} \mathrm{C}\end{array}$} & \multirow{2}{*}{$\begin{array}{l}\text { Mol. Form. } \\
\text { (Mol. Wt.) }\end{array}$} & \multicolumn{3}{|c|}{$\begin{array}{l}\text { Analysis }(\%) \\
\text { Calcd./Found }\end{array}$} \\
\hline & & & & & & $\mathrm{C}$ & $\mathrm{H}$ & $\mathrm{N}$ \\
\hline $6 a$ & $\mathrm{CH}_{3}$ & 22 & $14 \mathrm{~h}$ & $294-6$ & $\begin{array}{l}\mathrm{C}_{18} \mathrm{H}_{17} \mathrm{~N}_{7} \mathrm{O}_{4} \\
(395.3)\end{array}$ & $\begin{array}{l}54.68 \\
55.01 \\
\end{array}$ & $\begin{array}{l}4.30 \\
3.98 \\
\end{array}$ & $\begin{array}{l}24.81 \\
24.42 \\
\end{array}$ \\
\hline $6 b$ & $\mathrm{C}_{6} \mathrm{H}_{5}$ & 30 & $20 \mathrm{~h}$ & $310-2$ & $\begin{array}{l}\mathrm{C}_{23} \mathrm{H}_{19} \mathrm{~N}_{7} \mathrm{O}_{4} \\
(457.4)\end{array}$ & $\begin{array}{l}60.39 \\
59.90\end{array}$ & $\begin{array}{l}4.16 \\
4.55\end{array}$ & $\begin{array}{l}21.44 \\
20.96\end{array}$ \\
\hline
\end{tabular}

${ }^{1} \mathrm{H}$ NMR 6a: $\left(\mathrm{DMSO}_{6}\right)$ : $\delta 2.61\left(\mathrm{~s}, 3 \mathrm{H}, \mathrm{CH}_{3}\right), 3.77\left(\mathrm{~s}, 3 \mathrm{H}, \mathrm{NCH}_{3}\right), 3.89\left(\mathrm{~s}, 9 \mathrm{H}, 3 \mathrm{OCH}_{3}\right), 7.36(\mathrm{~s}$, $2 \mathrm{H}, \mathrm{ArH})$. MS of $\mathbf{6 a} ; \mathrm{m} / \mathrm{z} 395\left(\mathrm{M}^{+}\right)$; of $\mathbf{6 b} ; \mathrm{m} / \mathrm{z} 457\left(\mathrm{M}^{+}\right)$. IR $\left(\mathrm{cm}^{-1}\right)$ of $\mathbf{6 a}: 2232(\mathrm{C} \equiv \mathrm{N}), 1660$ $(\mathrm{C}=\mathrm{O})$, of 6a: $2211(\mathrm{C} \equiv \mathrm{N}), 1685(\mathrm{C}=\mathrm{O})$. 
Table 4. 6-Cyano-3-(3-methylpyrazol-1-yl)-8-methyl-5-(3,4,5-trimethoxyphenyl)-7,8-dihydro $[1,2,4]$ triazolo[4,3-a]pyrimidin-7-ones 10a,b

\begin{tabular}{|c|c|c|c|c|c|c|c|}
\hline \multirow{2}{*}{$\begin{array}{l}\text { Comp } \\
\text { d. No. }\end{array}$} & \multirow[t]{2}{*}{$\mathrm{R}$} & \multirow{2}{*}{$\begin{array}{l}\text { Yield } \\
\%\end{array}$} & \multirow{2}{*}{$\underset{{ }^{\circ} \mathrm{C} . \mathrm{p} .}{\mathrm{o}}$} & \multirow{2}{*}{$\begin{array}{l}\text { Mol. Form. } \\
\text { (Mol. Wt.) }\end{array}$} & \multicolumn{3}{|c|}{$\begin{array}{l}\text { Analysis }(\%) \\
\text { Calcd./Found }\end{array}$} \\
\hline & & & & & $\mathrm{C}$ & $\mathrm{H}$ & $\mathrm{N}$ \\
\hline $10 a$ & $\mathrm{CH}_{3}$ & 35 & $270-2$ & $\begin{array}{l}\mathrm{C}_{21} \mathrm{H}_{21} \mathrm{~N}_{7} \mathrm{O}_{4} \\
(435.4)\end{array}$ & $\begin{array}{l}57.93 \\
57.62\end{array}$ & $\begin{array}{l}4.83 \\
5.03\end{array}$ & $\begin{array}{l}22.53 \\
23.00\end{array}$ \\
\hline $10 b$ & $\mathrm{C}_{6} \mathrm{H}_{5}$ & 30 & $315-7$ & $\begin{array}{l}\mathrm{C}_{26} \mathrm{H}_{23} \mathrm{~N}_{7} \mathrm{O}_{4} \\
(497.5)\end{array}$ & $\begin{array}{l}62.77 \\
63.02 \\
\end{array}$ & $\begin{array}{l}4.62 \\
4.23 \\
\end{array}$ & $\begin{array}{l}19.72 \\
20.02 \\
\end{array}$ \\
\hline
\end{tabular}

IR $\left(\mathrm{cm}^{-1}\right)$ 10a, b: $2288(\mathrm{C} \equiv \mathrm{N}), 1679(\mathrm{C}=\mathrm{O}) .{ }^{1} \mathrm{H}$ NMR 10a: $\left(\mathrm{DMSO}_{6}\right): \delta 2.51\left(\mathrm{~s}, 3 \mathrm{H}, \mathrm{CH}_{3}\right), 2.53$ $\left(\mathrm{s}, 3 \mathrm{H}, \mathrm{CH}_{3}\right), 3.80\left(\mathrm{~s}, 3 \mathrm{H}, \mathrm{NCH}_{3}\right), 3.84\left(\mathrm{~s}, 2 \mathrm{H}, p-\mathrm{OCH}_{3}\right), 3.86\left(\mathrm{~s}, 6 \mathrm{H}, 2 m-\mathrm{OCH}_{3}\right), 6.28(\mathrm{~s}, 1 \mathrm{H}$, $=\mathrm{CH}), 7.37$ (s, 2H, ArH). MS of 10a; $\mathrm{m} / \mathrm{z} 435\left(\mathrm{M}^{+}\right)$.

\section{Preliminary antimicrobial screening}

Different bacteria and fungi were subjected to susceptibility testing on Muller-Hinton agar medium by the disc agar diffusion method. ${ }^{24}$ The antimicrobial activity of some of the newly synthesized compounds was tested against each of the mentioned strains using trimethoprim as reference (Table 1).

Overnight culture was streaked on the surface of Muller-Hinton agar plate. Sterile filter paper disc was saturated with $10 \mu \mathrm{l}$ of $0.5 \mathrm{mg} / \mathrm{ml} \mathrm{w} / \mathrm{v}$ solution of the compound under investigation in DMF. The plates and discs were then incubated at $37{ }^{\circ} \mathrm{C}$ for $24 \mathrm{~h}$ and examined for inhibition zones to determine the activity of the tested compounds. Control testing using DMF was used to determine the solvent effect.

\section{Acknowledgements}

The author would like to express their thanks to Professor Eman Mahmoud Hassan El-Masri, Microbiological Department, Faculty of Pharmacy, Zagazig University for measuring the antimicrobial activities.

\section{References and Notes}

1. Rane, N.; Gurram, V. K. Bioorg. Med. Chem. Lett. 2004, 14, 4185.

2. Prakash, O.; Bhardwaj, V.; Kuma, R.; Tyagi, P.; Areja, K. R. Eur. J. Med. Chem. 2004, 39, 1073.

3. Botta, M.; Artico, M.; Massa, S.; Gambacorta, A.; Maronigu, M. E.; Pani, A.; Lacolla, P. Eur. J. Med. Chem. 1992, 27, 251. 
4. Agarwal, N.; Srivastava, P.; Raghuwanshi, S. K.; Upadhyay, D. N.; Shukla, P. K.; Ram, V. J. Bioorg. Med. Chem. 2002, 10, 869.

5. Forsch, R. A.; Queener, S. F.; Rosowsky, A. Bioorg. Med. Chem. Lett. 2004, 14, 1811.

6. Agarwal, N.; Raghuwanshi, S. K.; Upadhyay, D. N.; Shukla, P. K.; Ram, V. J. Bioorg. Med. Chem. Lett. 2000, 10, 703.

7. Gillbert, M. D.; Wise, J.; Wainstein, M. D. S.; Goldberg, B. S. P.; Philip, M. D.; Kiozinn, J. Urology 1974, 3, 708.

8. Agarwal, N.; Srivastava, K.; Puvi, S. K.; Sinha, S.; Chauhan, P. M. S. Bioorg. Med. Chem. Lett. 2005, 15, 218.

9. Galina, G.; Tada, M.; Roumyana, G.; Evgeny, G. Arch. Cocology 2005, 13, 62.

10. Delia, T. J.; Baumann, M.; Bunker, A. Heterocycles 1993, 25, 1397.

11. Cocco, M. T.; Congin, C.; Lilliu, V.; Onnis, V. Bioorg. Med. Chem. 2006, 14, 366.

12. Malik, V.; Singh, P.; Kumar, S. Tetrahedron 2006, 62, 5944.

13. Fan, X.; Zhang, X.; Zhou, L.; Keith, K. A.; Kern, E. R.; Torrence, P. F. Bioorg. Med. Chem. Lett. 2006, 16, 3224.

14. Brideau, R. J.; Wolcott, J. A. Antimicrob. Agents Chemother. 1985, 28, 485.

15. Botta, M.; Occhionero, F.; Nicoletti, R.; Mastromarino, P.; Conti, C.; Magrini, M.; Saladino, R. Bioorg. Med. Chem. 1999, 7, 1925.

16. Srivastava, R.; Mishra, A.; Ratap, R.; Bhakuni, D. S.; Srimal, R. C. Thrombosis Res. 1989, 54, 741.

17. El-Ashmawy, M.; El-Feky, S. A.; El-Samii, Z. K.; Osman, N. A. Bull. Chim. Farm. 1997, $136,492$.

18. Skulnick, H. I.; Ludens, J. H.; Wendling, M. G.; Glenn, E. M.; Rohloff, N. A.; Smith, R. J.; Wierenga, W. J. Med. Chem. 1986, 29, 1499.

19. Atwal, K. S.; Swanson, B. N.; Unger, S. E.; Floyed, D. M.; Moreland, S.; Hedberg, A.; O'Reilly, B. C. J. Med. Chem. 1991, 34, 806.

20. Rovnyak, G. C.; Atwal, K. S.; Hedberg, A.; Kimball, S. D.; Moreland, S.; Gougoutas, J. Z.; O'Reilly, B. C.; Schwartz, J.; Marllery, M. F. J. Med. Chem. 1992, 35, 3254.

21. Grover, G. J.; Dzwonczyk, S.; McMullen, D. M.; Norandin, D. E.; Parham, C. S.; Sleph, P. G.; Moreland, S. J. Cardiovasc. Pharm. 1995, 26, 289.

22. Geies, A. A.; El-Dean, A. M. K.; Moustafa, O. Sh. Pharmazie 1997, 52, 436.

23. Kadry, A. M.; Abdel Aal, E. H.; Abdel Fattah, H. A.; Al-Mahmoudy, A. M. Bull. Fac. Pharm. Cairo Univ. 2005, 43, 243.

24. Eircsson, H. M.; Sherris, J. C. Acta Pathol. Microbial. Scand. B 1971, 217, 1. 